\title{
Heated Moisture Exchanger (HME) and dead space ventilation. Is Isocapnic conditions unachievable in children?
}

\section{To the Editor}

Pulmonary dead space under general paediatric anaesthesia is a useful and relevant parameter [1]. We know that Heated Moisture Exchanger (HME) may lead to larger dead space and can negatively impact mechanical ventilation and gas exchange $[1,2]$. Smaller HME appears to be preferable, but there is a lack of information regarding their applicability and suitability in paediatric population [2].

On this issue, Kwon [3] analyzed the effects of HME on dead space in 20 relatively healthy paediatric patients, during their exposure to general anaesthesia for minor surgical procedures. We would like to congratulate the authors on their original hypothesis and results, which have clinical crucial implications. They reported that $\mathrm{PaCO} 2$ was significantly influenced by HME and the difference was correlated with weight $(\mathrm{P}<0.001)$ and age $(\mathrm{P}=0.002)$ of a patient. However some major considerations and questions should be taken into consideration for the definitive clinical implications of their results.

Firstly, clinical consequences of the removal of HME for 15 minutes without compensatory use of another artificial humidification may lead to serious implications, such as cilia and endothelial injury, increase in airway resistance, hypothermia, and etc. $[2,4]$. We have not been provided with any information in regards to these well known consequences, as well as whether or not any attempt was made to measure such complications.

Secondly, there is insufficient information on the baseline of mechanical ventilation in both situations, which may influences our interpretation of higher pCO2 levels [4]. We have no information regarding the airway resistance, compliance, or peak airway pressure, which could help us for a better interpretation [5]. It would have been important to know the difference in $\mathrm{PaCO} 2$ between the baseline and after experimentation rather than just knowing the difference at the end of the experiment $[3,4]$. It is hard to determine whether or not the reduction is temporal and not a direct influence. Thirdly, main thrust of this report is on the alteration in physiological variables rather than clinically important outcomes, such as length mechanical ventilation or influence on weaning [5].

Overall, this report does not answer what are clinically important questions, such as which device is better for humidification, what effects of clinically important outcomes can be derived from this experiment and how best to use humidification without causing lung injury. In our opinion, this attractive and original report fails to even bridge a tile in this puzzle of humidification during healthy pediatric anesthesia.

\section{Antonio M. Esquinas ${ }^{1}$, and Prakesh S Shah ${ }^{2}$}

${ }^{1}$ Intensive Care Unit, Hospital Morales Meseguer, Murcia, Spain, ${ }^{2}$ Departments of Paediatrics and HPME, University of Toronto, Mount Sinai Hospital, Toronto, Canada

E-mail: antmesquinas@gmail.com

\section{References}

1. Monrigal JP, Granry JC. The benefit of using a heat and moisture exchanger during short operations in young children. Paediatr Anaesth 1997; 7: 295-300.

2. Nakagawa NK, Macchione M, Petrolino HM, Guimarães ET, King $\mathrm{M}$, Saldiva PH, et al. Effects of a heat and moisture exchanger and a heated humidifier on respiratory mucus in patients undergoing mechanical ventilation. Crit Care Med 2000; 28: 312-7.

3. Kwon MA. The effect of a pediatric heat and moisture exchanger on dead space in healthy pediatric anesthesia. Korean J Anesthesiol 2012; 62: 418-22.

4. Chiaranda M, Verona L, Pinamonti O, Dominioni L, Minoja G, Conti G. Use of heat and moisture exchanging (HME) filters in mechanically ventilated ICU patients: influence on airway flowresistance. Intensive Care Med 1993; 19: 462-6.

5. Girault C, Breton L, Richard JC, Tamion F, Vandelet P, Aboab J, et al. Mechanical effects of airway humidification devices in difficult to wean patients. Crit Care Med 2003; 31: 1306-11.

() This is an open-access article distributed under the terms of the Creative Commons Attribution Non-Commercial License (http:// creativecommons.org/licenses/by-nc/3.0/), which permits unrestricted non-commercial use, distribution, and reproduction in any medium, provided the original work is properly cited. 


\section{In Response}

We would like to appreciate to Dr. Esquinas and Shah for their interest regarding our study [1] that the use of heat and moisture exchanger (HME) in healthy pediatric patients significantly increases dead space ventilation.

In our study, 20 American Society of Anesthesiologists physical class I pediatric patients who underwent elective surgery under general anesthesia were enrolled. During the study, mean airway pressure, expiratory tidal volume and minute ventilation was not changed. Hemodynamic variables were consistently maintained, too. With the removal of HME for 15 minutes, EtCO2 and PaCO2 significantly decreased and the difference of $\mathrm{PaCO} 2$ with or without HME were significantly correlated to the weight and the age. If the study design were the comparison between independent groups with or without HME, the difference in PaCO2 between baseline and after experimentation would be clear to reveal it would be a temporal and not direct influence.

As mentioned in discussion, we did not use other compensatory humidification device during the period without HME, because the cessation time was short and we used a low gas flow. Even though our study did not contain the examination of injury in cilliary movements or endothelial injuries, the emergency and recovery were not prolonged or complicated in all cases. There was no significant coughing, hypothermia $<36^{\circ} \mathrm{C}$, desaturation $<90 \%$ or a progression to pneumonia.

Consequently, we revealed the use of HME significantly increased dead space ventilation even in the healthy pediatric patients. Further investigations should be needed to reveal which device would be better to maintain humidity in healthy pediatric patients, regarding the cost and the effect.

\section{MIn A Kwon}

Department of Anesthesiology and Pain Medicine, Dankook University Hospital, Cheonan, Korea

E-mail:mmauss@hanmail.net

\section{Reference}

1. Kwon MA. The effect of a pediatric heat and moisture exchanger on dead space in healthy pediatric anesthesia. Korean J Anesthesiol 2012; 62: 418-22. 